\title{
ASO Visual Abstract: Stage Migration and Survival Trends in Laryngeal Cancer
}

Michael M. Li, MD ${ }^{1}$, Songzhu Zhao, $\mathrm{MS}^{2}$, Antoine Eskander, MD, ScM ${ }^{3}$, Chandler Rygalski, $\mathrm{BS}^{4}$, Guy Brock, PhD $^{2}$, Anuraag S. Parikh, MD ${ }^{1}$, Catherine T. Haring, MD $^{1}$, Brian Swendseid, MD $^{1}$, Kevin Y. Zhan, MD ${ }^{1}$, Carol R. Bradford, $\mathrm{MD}^{1}$, Theodoros N. Teknos, $\mathrm{MD}^{5}$, Ricardo L. Carrau, MD, MBA ${ }^{1}$, Kyle K. VanKoevering, MD ${ }^{1}$, Nolan B. Seim, MD ${ }^{1}$, Matthew O. Old, $\mathrm{MD}^{\mathbf{1}}$, James W. Rocco, MD, PhD ${ }^{1}$, Sidharth V. Puram, MD, $\mathrm{PhD}^{6,7}$, and Stephen Y. Kang, $\mathrm{MD}^{1}$

${ }^{1}$ Department of Otolaryngology-Head and Neck Surgery, The Ohio State University Wexner Medical Center, Columbus, $\mathrm{OH} ;{ }^{2}$ Department of Biomedical Informatics and Center for Biostatistics, The Ohio State University, Columbus, $\mathrm{OH}$; ${ }^{3}$ Department of Otolaryngology-Head and Neck Surgery, Sunnybrook Health Sciences Center, Toronto, ON, Canada; ${ }^{4}$ College of Medicine, Ohio State University, Columbus, OH; ${ }^{5}$ University Hospital Seidman Cancer Center, Cleveland, $\mathrm{OH} ;{ }^{6}$ Department of Otolaryngology-Head and Neck Surgery, Washington University School of Medicine, St. Louis; ${ }^{7}$ Department of Genetics, Washington University School of Medicine, St. Louis

Although significant advances in treatment protocols were developed in the 2000s, laryngeal cancer survival did not improve from 2004 to 2016. Concurrently, stage migration toward greater proportions of stage IV disease has occurred. Early diagnosis and treatment are critical to optimize outcomes (https://doi.org/10.1245/s10434-021-10318-1).

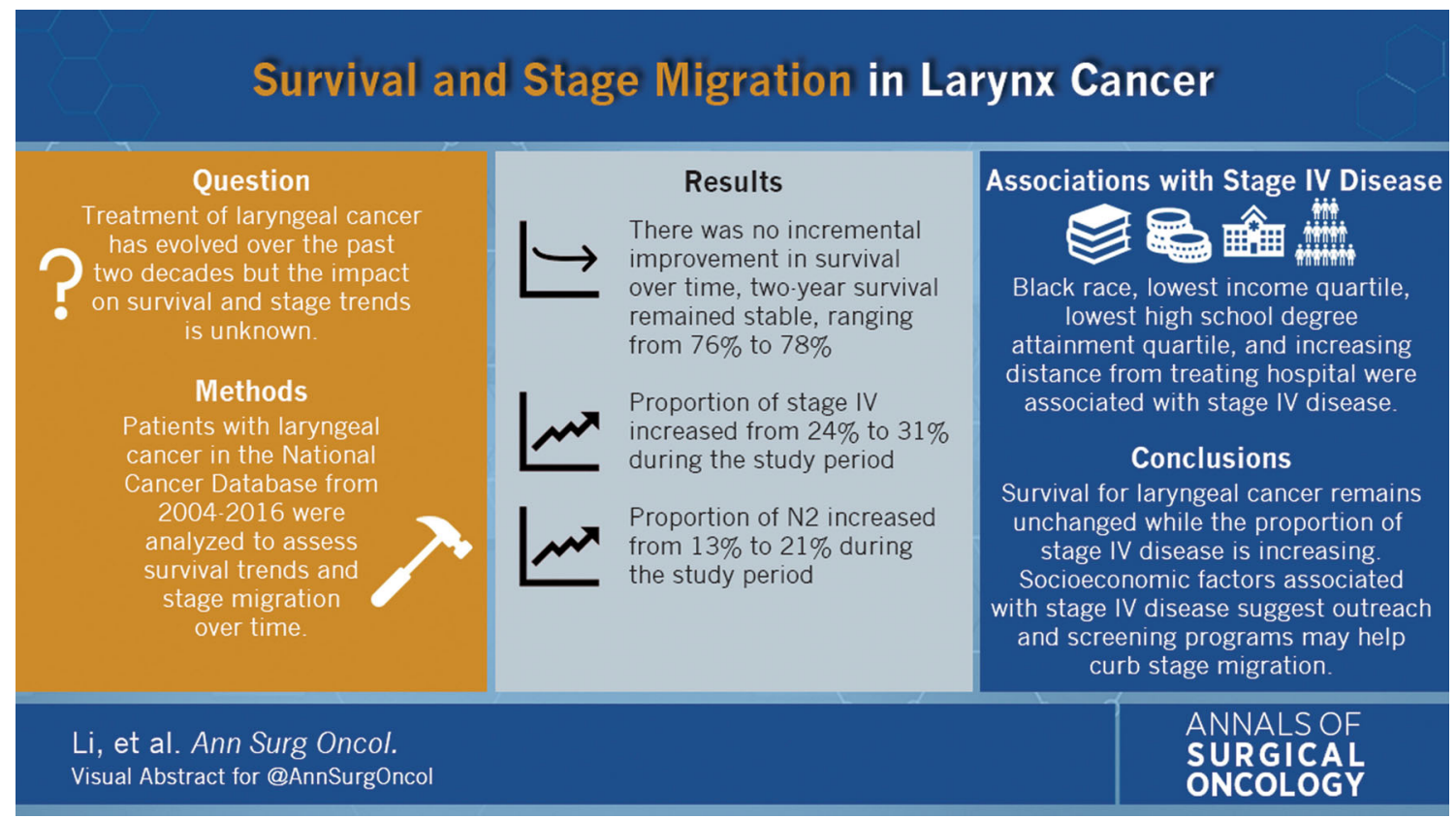

(C) Society of Surgical Oncology 2021

Publisher's NoteSpringer Nature remains neutral with regard to Published Online: 3 August 2021

S. Y. Kang, MD

e-mail: stephen.kang@osumc.edu 